\title{
Quantitation in Image Analysis: Practical Considerations for Drug Discovery
}

\author{
Michail A. Esterman* and Jeffrey C. Hanson \\ * Discovery Information Technology, Lilly Research Labs, Lilly Corporate Center Drop Code \\ 0545, Indianapolis, IN 46285
}

The traditional disciplines of science are grounded in the observation and measurement of object properties, static and dynamic. The development of imaging tools such as the microscope extended the range of natural vision and enabled scientist to observe, record and measure otherwise inaccessible object properties by means of their images. ${ }^{1}$ Over the last decade, the development of computer-based imaging systems resulted from technological advances in computer science and light detectors. These technologies have increased the number of object properties that can be observed as well as the accuracy and reproducibility with which they can be measured.

The biological sciences have entered the post-genomic era where the focus has shifted from cataloging the genetic complement to understanding how individual proteins participate in the biochemical pathways that govern cell and tissue functions. Imaging technology can be used for analysis of complex cellular properties, allowing phenotype, rather than the activity of individual targets, to drive lead generation. This approach aims to identify molecules that alter specific cellular properties, allowing identification of drugs that may act at a variety of points in functional target dependent and independent cellular pathways. ${ }^{2}$

Imaging deals with the principles, strategies and methods of image formation and the measurement of object properties which can be characterized by their numerical values. Imaging requires consideration of physics, biophysics, mathematics, statistics, computer science, and engineering. These disciplines must be applied to the physical system being imaged.

Digital imaging systems provide: Objective measure of spatial and temporal values, measurement of multiple variables in real time; programmatically and explicitly defined analysis; and sub population analysis, which can be crucial for defining the biological activity of a candidate compound. The ultimate goal of digital imaging in drug discovery is to convert digital representations, images, with multiple dimensions (size, shape, brightness, color,) to onedimensional data values, which can easily be tabulated, compared and understood.

In any scientific detection / measurement system, certain principles must be followed. For digital imaging, close attention must be paid to the principles involved in sample preparation, detection and acquisition, image enhancement, segmentation, and measurement of the experimental entity. These principles are illustrated by examples like object counting, surface area measurement, coefficient of co-localization, and protein compartmentalization.

1. Beck, R., 1993. Proc Natl Academy Sci USA. Vol 90: 9746-9750.

2. Trask, O.J. and Large, T. 2001. Current Drug Discovery, 
la

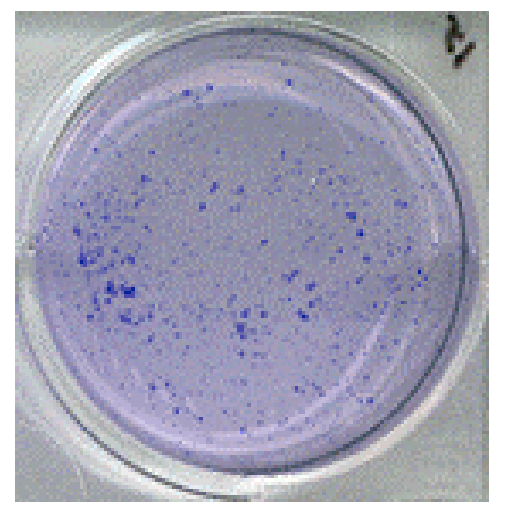

2a

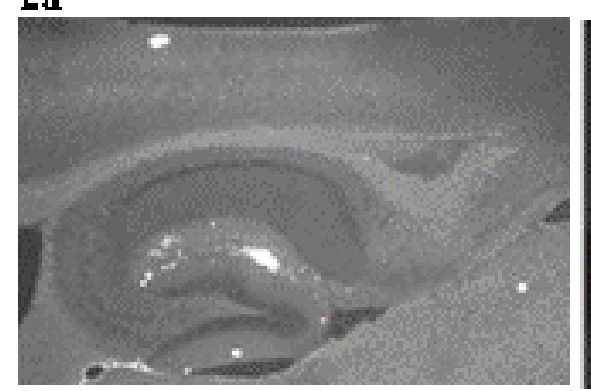

b

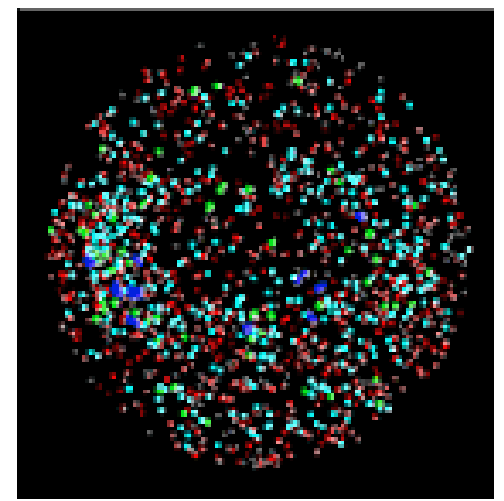

b

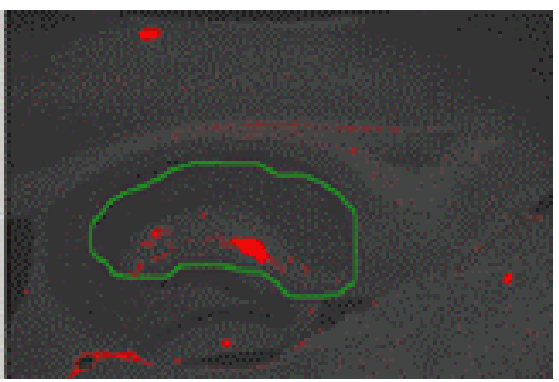

c

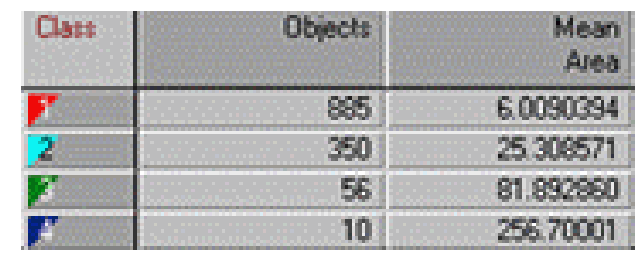

C

Total Area Plajue Area Playee Berden \begin{tabular}{l|l|r|}
54435 & 5019 & 0.092
\end{tabular}

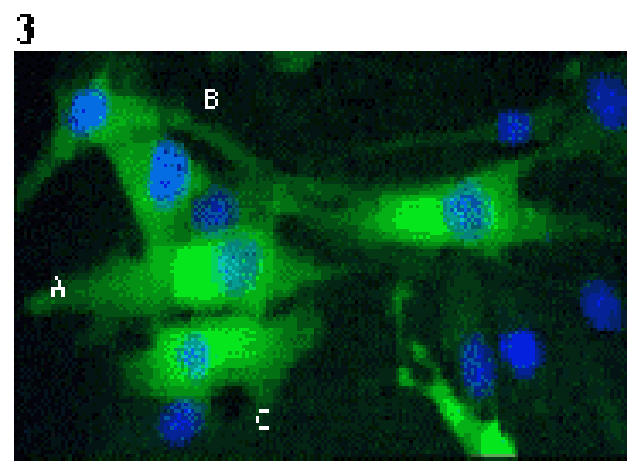

Figure legends

Figure 1. Image of cell colonies (a), image with interfering objects removed, analyzed and classified by colony size (b) and data table of count by size classification (c)

Figure 2. Image of mouse brain containing Beta Amyloid plaque (a), Region of interest and segmented plaque (b), and analysis results of surface area measurement.

Figure 3. Staining for receptor (green) and nuclei (blue) image demonstrates three sub populations of cells A strongly staining receptor, B lightly staining receptor and C cells with no receptor evident. 